\title{
Adiabatic nanofocusing in hybrid gap plasmon waveguides on the silicon-on-insulator platform
}

\author{
Michael P. Nielsen, Lucas Lafone, Aliaksandra Rakovich, Themistoklis P. H. Sidiropoulos, \\ Mohsen Rahmani, Stefan A. Maier, and Rupert F. Oulton
}

Department of Physics, Imperial College London, London, SW7 2AZ, UK

ABSTRACT: We present an experimental demonstration of a new class of hybrid gap plasmon waveguides on the silicon-on-insulator (SOI) platform. Created by the hybridization of the plasmonic mode of a gap in a thin metal sheet and the transverse-electric (TE) photonic mode of an SOI slab, this waveguide is designed for efficient adiabatic nanofocusing simply by varying the gap width. For gap widths greater than $100 \mathrm{~nm}$, the mode is primarily photonic in character and propagation lengths can be many tens of micrometers. For gap widths below $100 \mathrm{~nm}$, the mode becomes plasmonic in character with field confinement predominantly within the gap region and with propagation lengths of a few microns. We estimate the electric field intensity enhancement in hybrid gap plasmon waveguide tapers at $1550 \mathrm{~nm}$ by 3-photon absorption of selectively deposited CdSe/ZnS quantum dots within the gap. Here, we show electric field intensity enhancements of up to $167 \pm 26$ for a $24 \mathrm{~nm}$ gap, proving the viability of low loss adiabatic nanofocussing on a commercially relevant photonics platform.

KEYWORDS: nanofocusing, plasmonics, nano-optics, silicon photonics, nonlinear optics

Photonic integrated circuits have integration densities that are restricted by the weak nonlinear optical interactions necessary for electro-optic modulation and all-optical signal processing. Plasmonic waveguides will play a crucial role in the merger of photonics and electronics by combining speed at optical frequencies with chip-level device integation ${ }^{1}$. For example, plasmonic modulators with deep sub-wavelength optical confinement can be considerably smaller than their photonic counterparts ${ }^{2}$ by enhancing naturally weak nonlinear responses of materials ${ }^{3}$. The capability to focus optical signals far below the vacuum wavelength using plasmonics, to the scale of just tens of nanometres, provides a route to enhancing the weak optical nonlinearity in natural materials ${ }^{4}$ when short interaction lengths are required; e.g. within photonic systems where high device integration density is required. Indeed, extreme nanofocusing ${ }^{5}$ has been explored in antennas ${ }^{6-8}$ and waveguides $^{9-12}$ for sensing ${ }^{13}$ and nonlinear optics ${ }^{14,15}$; however, it remains unclear how existing photonics technologies ${ }^{16,17}$ can incorporate this capability. While extreme nanofocusing is now becoming recognized as a method to generate intense optical fields ${ }^{5,11,18}$, it still remains a challenge to move beyond proof-of-principle to technologically relevant implementations. The difficulty stems from the fact that semiconductor materials, used in established photonic technologies, exacerbate the loss inherent with plasmonic-based confinement ${ }^{19}$. And yet semiconductors have evolved in tandem with nanophotonics as they not only provide exceptional optoelectronic functionality but also the means to confine and route optical signals over a photonic chip.

This challenge of high ohmic loss in semiconductor plasmonics has been partially mitigated by the introduction of hybrid plasmonics ${ }^{20}$. Hybrid plasmonic waveguides incorporate a low index dielectric layer between the metal film and the high index semiconductor core region. This reduces propagation losses without sacrificing high modal confinement through the creation of a capacitor-like confinement mode in the low index dielectric region. Though hybrid plasmonic waveguides have been proposed for integration with silicon photonics ${ }^{21-24}$, obtaining and utilizing the strong confinement via nanofocusing is still a challenge. This letter presents an experimental demonstration of efficient adiabatic nanofocusing by a silicon hybrid gap plasmon waveguide ${ }^{25}$. The plasmonic waveguide is lithographically defined on a silicon-on-insulator (SOI) platform to achieve optical localisation within gaps as small as $24 \mathrm{~nm}$, comparable to gate sizes in current transistor technology. Photoluminescence of three-photon excited CdSe/ZnS quantum dots within the $24 \mathrm{~nm}$ gap determines the electric field intensity enhancement of $167 \pm 26$.

\footnotetext{
*r.oulton@imperial.ac.uk
} 

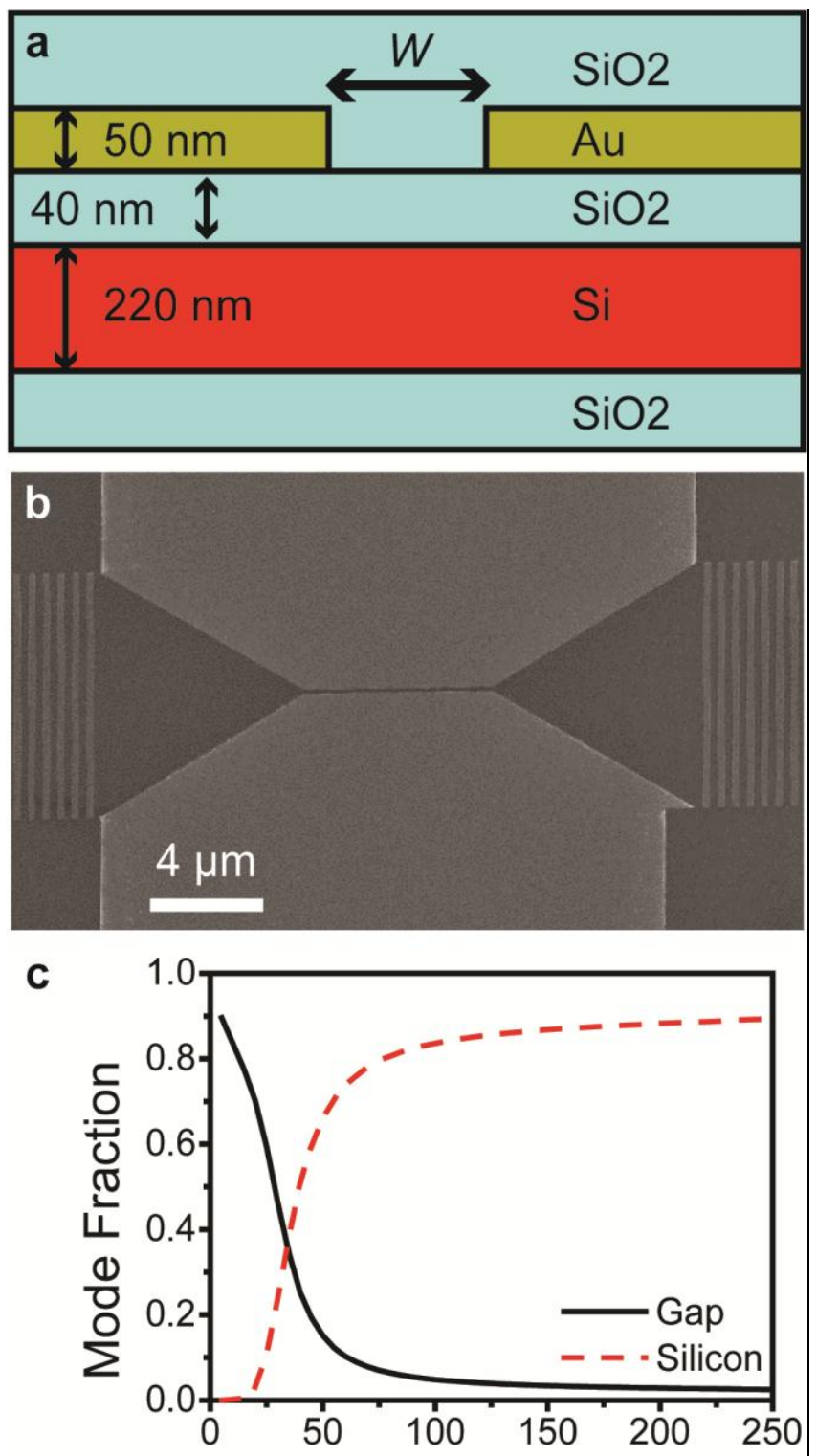

\section{Gap Width [nm]}

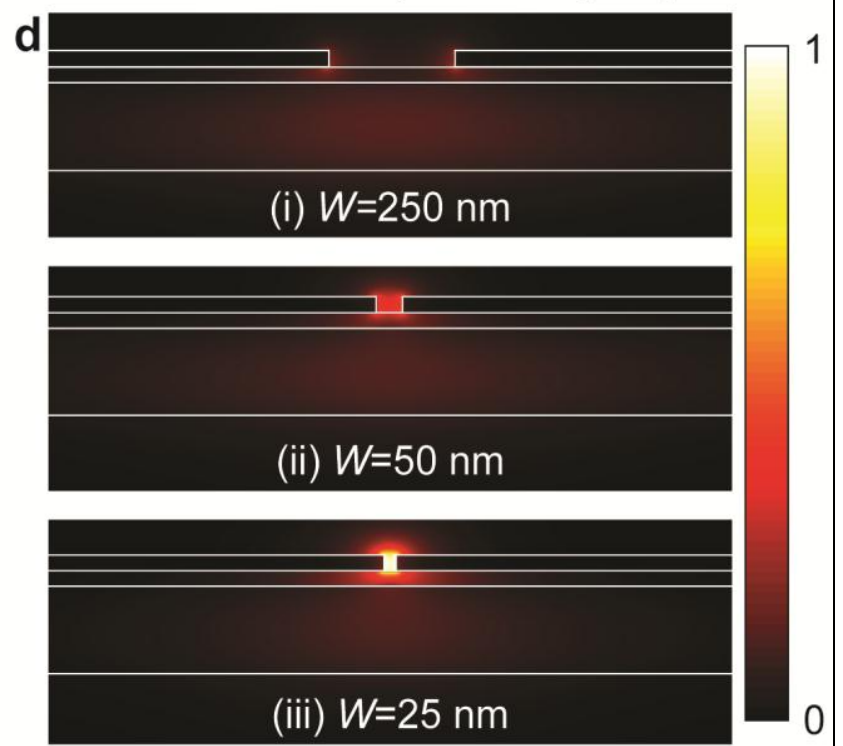

Figure 1. (a) Schematic of the demonstrated hybrid silicon gap plasmon waveguide. (b) SEM image of a $120 \mathrm{~nm}$ wide waveguide with coupling tapers and gratings. (c) Fraction of mode in either the gap 
or the silicon layer as a function of gap width. (d) Mode diagrams at (i) $250 \mathrm{~nm}$, (ii) $50 \mathrm{~nm}$, and (iii) 25 $\mathrm{nm}$ wide gap widths showing the crossover from a primarily photonic mode to a primarily plasmonic mode. All three mode diagrams share the same colour scale bar. Due to the high field concentration in the gap at $W=25 \mathrm{~nm}$, the scale bar saturates the field in the $25 \mathrm{~nm}$ gap in order to improve visibility of the modes.

A recent theoretical proposal showed that these hybrid gap plasmonic waveguides had unique advantages for nanofocusing applications ${ }^{25}$, along with long propagation lengths for large gap widths. At large gap widths, in excess of $100 \mathrm{~nm}$, the mode propagates primarily in the underlying silicon away from the metallic films, leading to propagation lengths of tens or even hundreds of micrometres ${ }^{25}$. When the waveguide width is reduced below $100 \mathrm{~nm}$, the mode becomes concentrated in the gap, leading to sub-wavelength confined modes and allowing for extreme nanofocusing. This provides a new degree of freedom in waveguide dispersion engineering as the localisation of the mode is purely controlled by the gap width. Moreover, one can imagine filling the gap region with a functional nonlinear material and, with the metal films acting as natural electrical contacts, creating a plasmonic phase $\mathrm{e}^{26,27}$ or absorption ${ }^{28}$ modulator. Moreover, the structure has also been shown to have promise theoretically for all-optical third order nonlinear effects such as four-wave mixing ${ }^{29}$.

A schematic of the demonstrated silicon hybrid gap plasmon waveguide can be seen in Figure 1a. The waveguide core is composed of a standard SOI wafer comprising a $220 \mathrm{~nm}$ device layer and a $3 \mu \mathrm{m}$ buried oxide layer with $40 \mathrm{~nm}$ of $\mathrm{SiO} 2$ and $40 \mathrm{~nm}$ of Au on top. An example of the fabricated structure can be seen in Figure 1b. Here $120 \mathrm{~nm}$ wide waveguides are accessed from free space through a simple grating coupler designed to couple into the TE mode of the underlying silicon slab waveguide. The grating coupler is comprised of Au strips with a $500 \mathrm{~nm}$ period and $50 \%$ fill factor. The advantage of these simple metal grating in-/out-couplers is that they can be fabricated along with the rest of the structure in a single lithographic step. Then a $30^{\circ}$ taper focuses the waveguide from the original width of $8 \mu \mathrm{m}$ at the grating couplers to the final waveguide width.

The primary advantage of this hybrid silicon plasmonic waveguide geometry over its counterparts $^{21-24}$ is the transition of the mode from a photonic mode in the silicon layer to a plasmonic mode in the gap region when the gap width reaches a critical point. This critical width is determined by the spacer thickness as well as the relative refractive indices of the waveguide core, and the spacer/cladding materials. For the waveguide described here, this occurs around $W \approx 50 \mathrm{~nm}$. In Fig. $1 \mathrm{c}$ we compare the fraction of the mode energy in the silicon and the gap region as a function of gap width, clearly illustrating the crossover. This crossover between photonic and plasmonic characteristics is accompanied by a rapid change in effective mode index, which can have consequences for adiabatic nanofocusing ${ }^{11}$ at gap widths of less than $20 \mathrm{~nm}^{25}$. Figure $1 \mathrm{~d}$ shows the mode distribution at three separate gap widths: (i) $250 \mathrm{~nm}$, (ii) $50 \mathrm{~nm}$, and (iii) $25 \mathrm{~nm}$. This is another visual demonstration of this crossover phenomena as the mode is primarily photonic at $W=250 \mathrm{~nm}$, then at $W \approx 50 \mathrm{~nm}$ there is a hybridization between the plasmonic and photonic modes, and then finally at $W=25 \mathrm{~nm}$ the mode is almost entirely plasmonic in character. If one was to consider changing the core material from silicon to a lower index photonic material such as $\mathrm{Si}_{3} \mathrm{~N}_{4}$, not only would this move the critical point to wider gap widths, but it will enable the structure to be used in the visible regime as well.

In order to show the usefulness of this waveguide geometry for both nanofocusing and lowloss waveguiding, waveguides with gaps ranging from $25 \mathrm{~nm}$ to $450 \mathrm{~nm}$ were fabricated. The cut-back method $^{30}$ was used to obtain the propagation lengths for each of the waveguide widths. Several waveguides of the same length were measured, with lengths ranging from $0 \mu \mathrm{m}$ (just the coupling tapers) all the way up to $50 \mu \mathrm{m}$ for the wider gap widths. An example of this can be seen in Figure $2 \mathrm{a}$, which shows the measured transmission at $1550 \mathrm{~nm}$ for the $25 \mathrm{~nm}$ wide waveguides and the resulting exponential fit. Figure $2 \mathrm{~b}$ depicts the results for all the fabricated devices along with the theoretical values. The experimental results agree quite closely with the theoretical values, especially considering the inherent roughness of the fabricated Au films. Additional details of the experiment can be found in the Supporting Information along with details on the theoretical calculations using 
COMSOL. Broadband white light measurements of the grating coupler efficiency and waveguide propagation lengths can also be found in the Supporting Information.

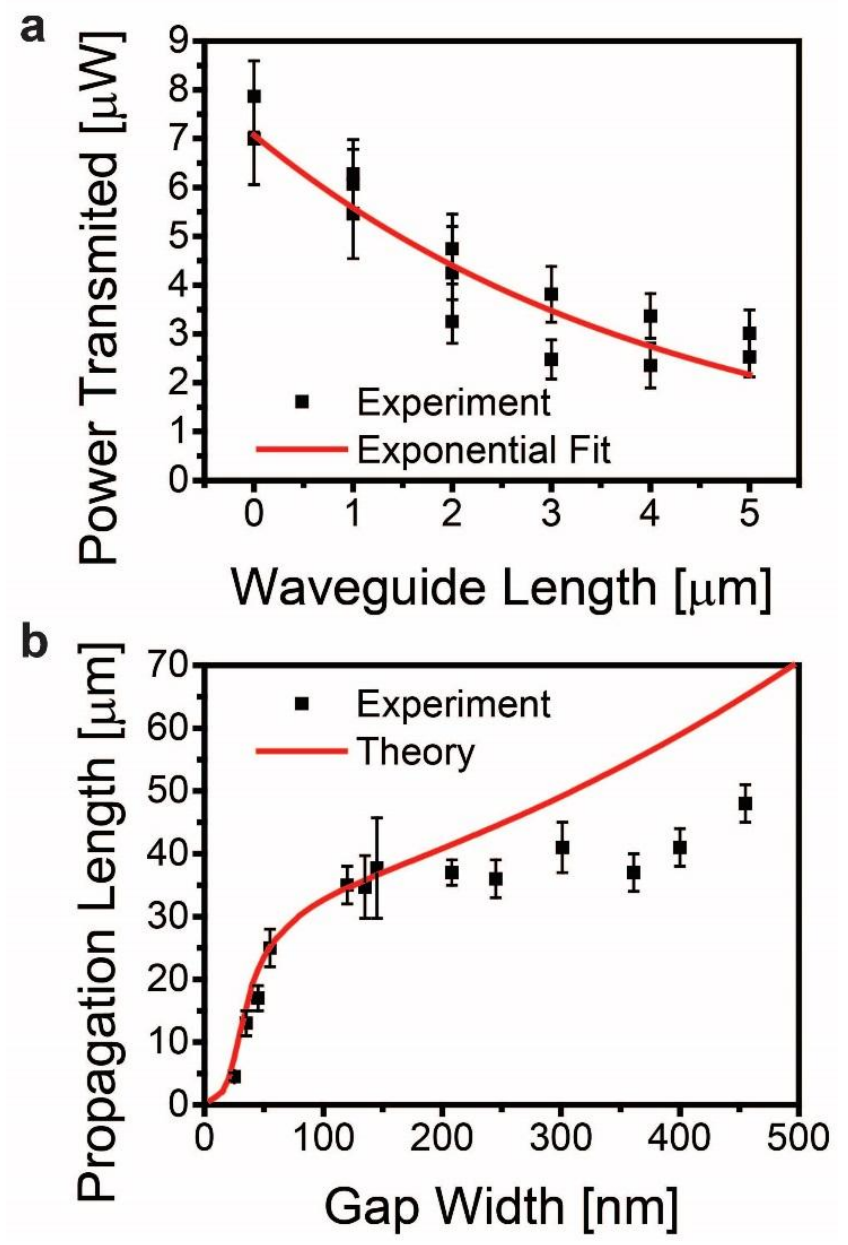

Figure 2. (a) An example of using cut-back method used to determine the propagation length of waveguides with gap widths of $25 \mathrm{~nm}$ from an exponential fit to the measured transmission through a number of waveguides of different lengths at $\lambda=1550 \mathrm{~nm}$. (b) Measured propagation lengths with the cut-back method compared to theoretical calculations.

Since one of the primary advantages of this class of hybrid plasmonic waveguides is its direct low-loss nanofocusing from a silicon photonic waveguide, by filling the gap region with a nonlinear fluorescent material one can directly measure the electric field enhancement inherent in the structure. In order to maximize the proportion of mode in the gap region, new samples were fabricated with the $\mathrm{SiO}_{2}$ spacer layer reduced to $30 \mathrm{~nm}$. Quantum dots (QDs) were then selectively deposited around the gratings, taper, and waveguide region through the use of a self-assembled monolayer as an adhesion layer in order to compare the fluorescent emission along the entire structure. Here, $655 \mathrm{~nm}$ emitting $\mathrm{COOH}$-functionalised CdSe/ZnS core-shell QDs were used as they can absorb the $1550 \mathrm{~nm}$ signal through a three photon absorption process and emit away from the second and third harmonic of the signal, which could be produced in either the silicon or metal layers. Details of the absorbance and photoluminescence of the QDs, along with details of the fabrication process, can be found in the Supporting Information. Figure 3a shows the final result of this process for a $5 \mu \mathrm{m}$ long waveguide without the cladding layer. It is possible to see the contrast depicting where the QDs were deposited, especially in the taper region compared to the surrounding non-metalized regions. In the inset, a close-up view of a $38 \mathrm{~nm}$ wide waveguide shows the individual QDs inside the gap region. 

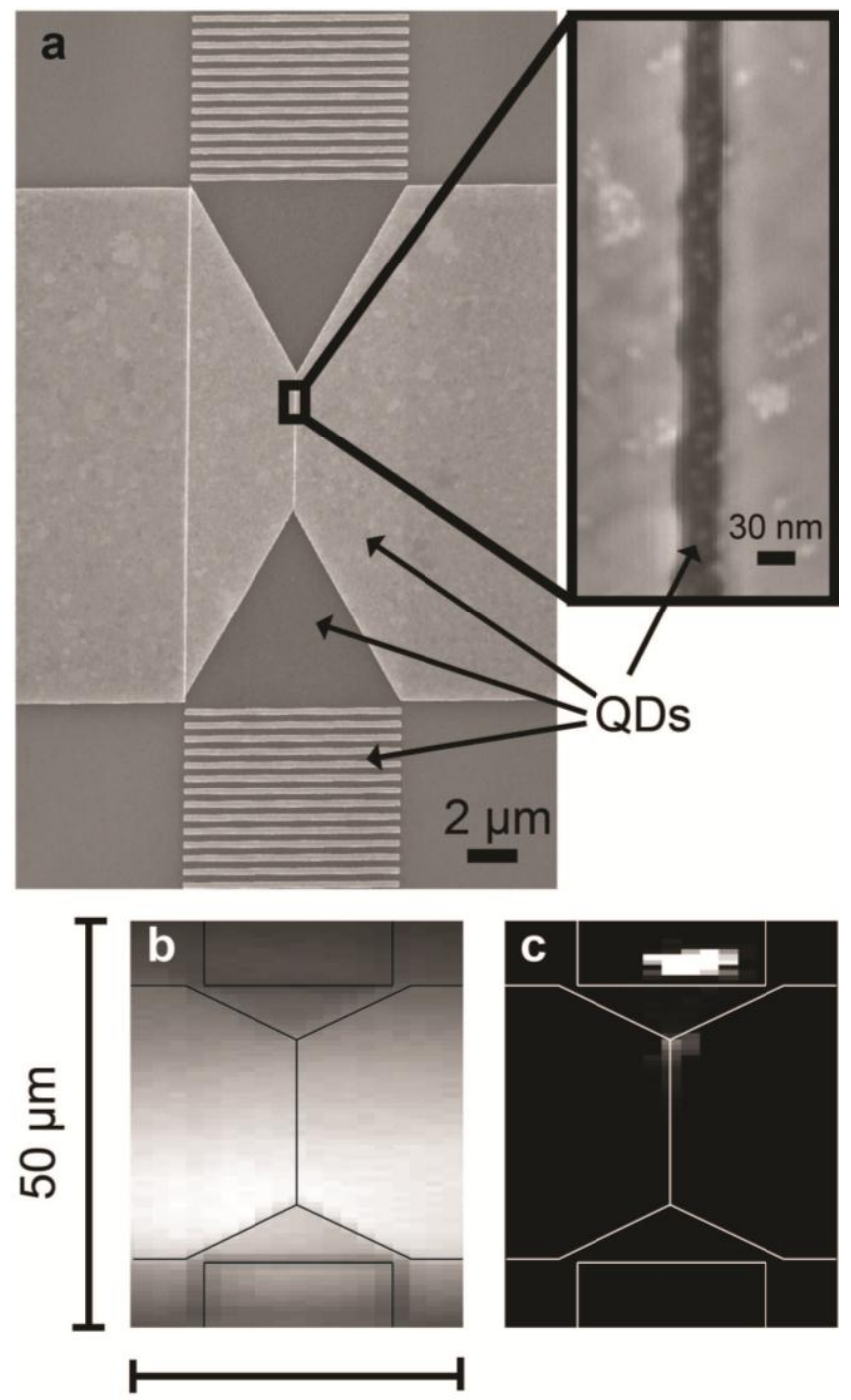

$15 \mu \mathrm{m}$

Figure 3. (a) SEM image of a $5 \mu \mathrm{m}$ long waveguide after selective deposition of quantum dots on the gratings, taper, and waveguide. The inset shows a close up view of the quantum dots in a $38 \mathrm{~nm}$ wide waveguide. (b) A 2-D LN-cooled Si spectrometer image of a $20 \mu \mathrm{m}$ long, $38 \mathrm{~nm}$ wide waveguide where each pixel is $750 \times 750 \mathrm{~nm}^{2}$. (c) Quantum dot photoluminescence filtered between $600-700 \mathrm{~nm}$ light from the same waveguide when $1550 \mathrm{~nm}$ light is focused on the top input grating as measured by the spectrometer. Guides to the eye have been drawn to delimitate the underlying structure.

In order to measure the electric field intensity enhancement arising from the nanofocusing of the hybrid plasmonic waveguide structure, the reflected and emitted light from the structure is imaged on a 2-D Si spectrometer. Figure $3 \mathrm{~b}$ depicts a white light image of a $20 \mu \mathrm{m}$ long, $38 \mathrm{~nm}$ wide waveguide on the spectrometer. Then, in Figure 3c, a spectrometer image is shown of the same waveguide when the $1550 \mathrm{~nm}$ input beam is aligned to the top input grating. Figures $3 \mathrm{~b}$ and $3 \mathrm{c}$ have been aligned in order to show the correlation of spatial locations of the QD emission. One can see emission at the input grating arising from the QDs distributed there. This fades at the start of the taper region due to the low mode overlap of a silicon photonic TE mode with the QDs atop the spacer layer, before rapidly growing stronger at the end of the taper as more of the mode becomes drawn into the gap region containing the QDs. From the end of the taper and start of the narrowest part of the waveguide, the emission drops off exponentially along the waveguide as expected due to the propagation loss. To give a better view of this emission, Figure 4 a shows the maximum pixel counts along $24 \mathrm{~nm}$ and $38 \mathrm{~nm}$ wide waveguide structures from top to bottom, with $0 \mu \mathrm{m}$ set at the start of the waveguide and the in-coupling grating located at $-10 \mu \mathrm{m}$. The three-photon luminescence peak 
from the QDs dispersed across the grating region differs sharply between the two waveguides due to the difference in input power and measurement integration time: $40 \mathrm{~mW}$ integrated over $60 \mathrm{~s}$ for the $24 \mathrm{~nm}$ wide waveguide and $80 \mathrm{~mW}$ integrated over $600 \mathrm{~s}$ for the $38 \mathrm{~nm}$ wide waveguide. Since the absorption of the $1550 \mathrm{~nm}$ signal is a third order process, the exponential decay constant of the QD emission along the waveguide is one third of the propagation length of the waveguide. Figure $4 \mathrm{~b}$ shows the extracted propagation lengths using this method for waveguides of $24 \mathrm{~nm}, 38 \mathrm{~nm}$, and 55 $\mathrm{nm}$ gap widths compared to the calculated values. There is very good correlation between the experimental and theoretical values in this case, better even than those obtained through the cut-back method depicted in Figure $2 \mathrm{~b}$. Here, for a $24 \mathrm{~nm}$ wide waveguide, we measured a propagation length of $5 \pm 1 \mu \mathrm{m}$ compared to a theoretical value of $5.3 \mu \mathrm{m}$. By comparing the maximum QD emission at the input grating against the start of the waveguide, one can estimate the normalized electric field intensity enhancement from the adiabatic nanofocusing from the start to end of the taper, in this case along a $30^{\circ}$ taper from an initial width of $8 \mu \mathrm{m}$, through Equation (1).

$$
E F=\left|\frac{I}{I_{o}}\right|=\left(\frac{C}{C_{o}} * \frac{W_{\text {pixel }}}{W}\right)^{1 / 3}\left(\frac{\rho_{Q D}(W)}{\rho_{Q D}\left(W_{o}\right)}\right) / \eta_{\text {grating }}
$$

Where $E F=\left|I / I_{o}\right|$ is the normalized electric field intensity enhancement or enhancement factor, $C$ is the spectrometer counts at the start of the waveguide, $C_{o}$ is the maximum spectrometer counts at the input grating, $W_{\text {pixel }}=750 \mathrm{~nm}$ is the width of a spectrometer pixel in the image, $W$ is the waveguide width, $\rho_{Q D}(W)$ is the density of QDs in the waveguide (see Supporting Information), $\rho_{Q D}\left(W_{o}\right)$ is the density of QDs at the input grating, and $\eta_{\text {grating }}$ is the grating coupling efficiency at $1550 \mathrm{~nm}$. The cubic root arises from this being a third order process in intensity. Figure $4 \mathrm{~b}$ also compares the experimentally determined normalized electric field intensity enhancement for waveguides of widths $24 \mathrm{~nm}, 38 \mathrm{~nm}$, and $55 \mathrm{~nm}$ to tapering simulations. Details of the tapering simulations can be found in the Supporting Information. Again, there is reasonable agreement between the experimental and theoretical results, with the experimental electric field intensity enhancement consistently below that of the simulated values. This is likely caused by the higher propagation losses in the experiment due to the rough metal films. The higher propagation losses also had a strong effect on the taper coupling efficiency, which was measured to be $65 \pm 10 \%$, significantly less than the predicted $95 \%$. For the 24 $\mathrm{nm}$ waveguide, we measured an electric field intensity enhancement of $167 \pm 26$ compared to a simulated electric field intensity enhancement of 144 . At these small waveguide widths, the electric field intensity enhancement is strongly dependent on the gap width, with just nanometer variations in gap width giving rise to enhancement fluctuations consistent with our experimental errors. Theoretically, below $24 \mathrm{~nm}$ gap widths the enhancement factor rapidly increases, approaching 1600 at a gap of $10 \mathrm{~nm}$ when adiabatic nanofocusing begins to break down ${ }^{25}$.

The presented silicon hybrid gap plasmon waveguide straddles the divide between low-loss conventional silicon photonic waveguides and high confinement plasmonic gap waveguides. Requiring only simple metal patterning, this new class of plasmonic waveguides is formed only of a thin gap in a metal film spaced above of a SOI substrate. This strategy is also compatible with the use of TE waveguide modes in standard silicon photonics ${ }^{31}$. For gap widths greater than $100 \mathrm{~nm}$, the mode is primarily photonic in character and propagates many tens of micrometres in the silicon layer, enabling efficient coupling to conventional TE silicon waveguides. As the gap width is tightened below $100 \mathrm{~nm}$, the mode becomes concentrated in the gap region. We have shown that this results in extreme nanofocusing of the electromagnetic energy of the mode, up to an electric field intensity enhancement of $167 \pm 26$ at a gap width of $24 \mathrm{~nm}$ compared to the original silicon nanophotonic mode. This could conceivable be improved significantly with the capability to manufacture gap size in the 10 $\mathrm{nm}$ range. Such extreme nanofocusing should give rise to efficient nonlinear processes, which in turn could lead to a dramatic decrease in required device length scales for nonlinear modulators, a requirement for achieving high device integration densities in photonic integrated circuits. 
a

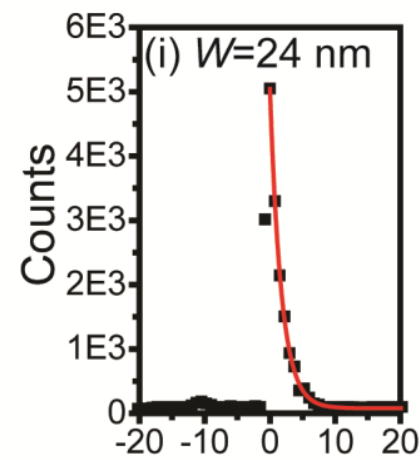

b

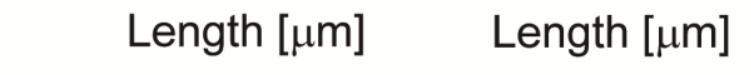

Figure 4. (a) Maximum pixel counts down the $20 \mu \mathrm{m}$ long, (i) $24 \mathrm{~nm}$ wide waveguide (40 $\mathrm{mW}$ input power integrated over $60 \mathrm{~s}$ ) and (ii) $38 \mathrm{~nm}$ wide waveguide (80 mW input power integrated over 600 s) with exponential fits to the QD emission decay spatially along the waveguides. (b) Extracted waveguide properties using nonlinear absorption and fluorescent emission from quantum dots in the gap waveguides depicting normalized enhancement factor $(E F)$ and propagation length $\left(L_{p}\right)$ at 1550 $\mathrm{nm}$ compared to calculated values.

\section{Supporting Information}

Details on the fabrication procedure, white light and linear waveguide characterisation, numerical simulations, quantum dot characterisation, and nonlinear experiments are included in a supporting information file.

\section{Acknowledgements}

The authors would like to thank Javier Cambiasso for assistance with the quantum dot functionalization and deposition. This work was sponsored by the Natural Sciences and Engineering Research Council of Canada (NSERC), Leverhulme Trust, and the EPSRC Reactive Plasmonics Programme (EP/M013812/1). S.M. acknowledges the Royal Society. R.F.O. is supported by an EPSRC Fellowship (EP/I004343/1) and Marie Curie IRG (PIRG08-GA-2010-277080).

\section{References}

(1) Ozbay, E. Plasmonics: Merging Photonics and Electronics at Nanoscale Dimensions. Science 2006, 311, 189-193.

(2) Reed, G. T.; Mashanovich, G.; Gardes, F. Y.; Thomson, D. J. Silicon Optical Modulators. Nat. Photonics 2010, 4, 518-526.

(3) MacDonald, K. F.; Zheludev, N. I. Active Plasmonics: Current Status. Laser Photon. Rev. 2009, 4, 562-567.

(4) Boyd, R. W. Nonlinear Optics; 3rd ed.; Academic Press: London, 2008.

(5) Gramotnev, D. K.; Bozhevolnyi, S. I. Nanofocusing of Electromagnetic Radiation. Nat. Photonics 2013, 8, 13-22. 
(6) Sidiropoulos, T. P. H.; Nielsen, M. P.; Roschuk, T. R.; Zayats, A. V.; Maier, S. A.; Oulton, R. F. Compact Optical Antenna Coupler for Silicon Photonics Characterized by Third-Harmonic Generation. ACS Photonics 2014, 1, 912-916.

(7) Aouani, H.; Rahmani, M.; Navarro-Cía, M.; Maier, S. A. Third-Harmonic-Upconversion Enhancement from a Single Semiconductor Nanoparticle Coupled to a Plasmonic Antenna. Nat. Nanotechnol. 2014, 9, 290-294.

(8) Kim, S.; Jin, J.; Kim, Y.-J.; Park, I.-Y.; Kim, Y.; Kim, S.-W. High-Harmonic Generation by Resonant Plasmon Field Enhancement. Nature 2008, 453, 757-760.

(9) Choo, H.; Kim, M.-K.; Staffaroni, M.; Seok, T. J.; Bokor, J.; Cabrini, S.; Schuck, P. J.; Wu, M. C.; Yablonovitch, E. Nanofocusing in a Metal-insulator-metal Gap Plasmon Waveguide with a Three-Dimensional Linear Taper. Nat. Photonics 2012, 6, 838-844.

(10) Desiatov, B.; Goykhman, I.; Levy, U. Plasmonic Nanofocusing of Light in an Integrated Silicon Photonics Platform. Opt. Express 2011, 19, 13150-13157.

(11) Stockman, M. Nanofocusing of Optical Energy in Tapered Plasmonic Waveguides. Phys. Rev. Lett. 2004, 93, 137404.

(12) Schnell, M.; Alonso-González, P.; Arzubiaga, L.; Casanova, F.; Hueso, L. E.; Chuvilin, A.; Hillenbrand, R. Nanofocusing of Mid-Infrared Energy with Tapered Transmission Lines. Nat. Photonics 2011, 5, 283-287.

(13) Anker, J. N.; Hall, W. P.; Lyandres, O.; Shah, N. C.; Zhao, J.; Van Duyne, R. P. Biosensing with Plasmonic Nanosensors. Nat. Mater. 2008, 7, 442-453.

(14) Park, I.-Y.; Kim, S.; Choi, J.; Lee, D.-H.; Kim, Y.-J.; Kling, M. F.; Stockman, M. I.; Kim, S.-W. Plasmonic Generation of Ultrashort Extreme-Ultraviolet Light Pulses. Nat. Photonics 2011, 5, 677-681.

(15) Sederberg, S.; Elezzabi, A. Y. Ponderomotive Electron Acceleration in a Silicon-Based Nanoplasmonic Waveguide. Phys. Rev. Lett. 2014, 113, 167401.

(16) Rukhlenko, I. D.; Premaratne, M.; Agrawal, G. P. Nonlinear Propagation in Silicon-Based Plasmonic Waveguides from the Standpoint of Applications. Opt. Express 2011, 19, 206-217.

(17) Krasavin, A. V; Zayats, A. V. Silicon-Based Plasmonic Waveguides. Opt. Express 2010, 18, 11791-11799.

(18) Zenin, V. A.; Andryieuski, A.; Malureanu, R.; Radko, I. P.; Volkov, V. S.; Gramotnev, D. K.; Lavrinenko, A. V; Bozhevolnyi, S. I. Boosting Local Field Enhancement by on-Chip Nanofocusing and Impedance-Matched Plasmonic Antennas. Nano Lett. 2015.

(19) Maier, S. A. Plasmonics: Fundamentals and Applications; 1st ed.; Springer: Bath, 2007.

(20) Oulton, R. F.; Sorger, V. J.; Genov, D. A.; Pile, D. F. P.; Zhang, X. A Hybrid Plasmonic Waveguide for Subwavelength Confinement and Long-Range Propagation. Nat. Photonics 2008, 2, 496-500.

(21) Nielsen, M. P.; Ashfar, A.; Cadien, K.; Elezzabi, A. Y. Plasmonic Materials for Metal-insulatorsemiconductor-insulator-metal Nanoplasmonic Waveguides on Silicon-on-Insulator Platform. Opt. Mater. 2013, 36, 294-298.

(22) Kwon, M.-S.; Shin, J.-S.; Shin, S.-Y.; Lee, W.-G. Characterizations of Realized Metal-InsulatorSilicon-Insulator-Metal Waveguides and Nanochannel Fabrication via Insulator Removal. Opt. Express 2012, 20, 21875-21887.

(23) Nielsen, M. P.; Elezzabi, A. Y. Nanoplasmonic Distributed Bragg Reflector Resonators for Monolithic Integration on a Complementary Metal-Oxide-Semiconductor Platform. Appl. Phys. Lett. 2013, 103, 051107.

(24) Goykhman, I.; Desiatov, B.; Levy, U. Experimental Demonstration of Locally Oxidized Hybrid Silicon-Plasmonic Waveguide. Appl. Phys. Lett. 2010, 97, 141106.

(25) Lafone, L.; Sidiropoulos, T. P. H.; Oulton, R. F. Silicon-Based Metal-Loaded Plasmonic Waveguides for Low-Loss Nanofocusing. Opt. Lett. 2014, 39, 4356-4359.

(26) Melikyan, A.; Alloatti, L.; Muslija, A.; Hillerkuss, D.; Schindler, P. C.; Li, J.; Palmer, R.; Korn, D.; Muehlbrandt, S.; Van Thourhout, D.; Chen, B.; Dinu, R.; Sommer, M.; Koos, C.; Kohl, M.; Freude, W.; Leuthold, J. High-Speed Plasmonic Phase Modulators. Nat. Photonics 2014, 8, 229-233.

(27) Zhu, S.; Lo, G. Q.; Kwong, D. L. Phase Modulation in Horizontal Metal-Insulator-SiliconInsulator-Metal Plasmonic Waveguides. Opt. Express 2013, 21, 8320-8330.

(28) Lee, H. W. H.; Papadakis, G.; Burgos, S. P.; Chander, K.; Kriesch, A.; Pala, R.; Peschel, U.; Atwater, H. A. Nanoscale Conducting Oxide PlasMOStor. Nano Lett. 2014, 14, 6463-6468.

(29) Duffin, T. J.; Nielsen, M. P.; Diaz, F.; Palomba, S.; Maier, S. A.; Oulton, R. F. Degenerate Four-Wave Mixing in Silicon Hybrid Plasmonic Waveguides. Opt. Lett. 2016, 41, 155-158. 
(30) Keck, D. B.; Tynes, R. Spectral Response of Low-Loss Optical Waveguides. Appl. Opt. 1972, 11, 1502-1506.

(31) Silicon Photonics II; Lockwood, D. J.; Pavesi, L., Eds.; 1st ed.; Springer: Berlin, 2011. 


\section{For Table of Contents Use Only}

\section{Adiabatic nanofocusing in hybrid gap plasmon waveguides on the silicon-on-insulator platform}

Michael P. Nielsen, Lucas Lafone, Aliaksandra Rakovich, Themistoklis P. H. Sidiropoulos, Mohsen Rahmani, Stefan A. Maier, and Rupert F. Oulton

Department of Physics, Imperial College London, London, SW7 2AZ, UK

While extreme nanofocusing is becoming a recognized method to generate intense optical fields, it still remains a challenge to move beyond proof-of-principle to technologically relevant implementations. The difficulty stems from the fact that semiconductor materials, used in established photonic technologies, exacerbate the loss inherent with plasmonic-based confinement. And yet semiconductors have evolved in tandem with nanophotonics as they not only provide optoelectronic functionality but also the means to confine and route optical signals over a photonic chip. In this letter, we demonstrate a nanofocusing device integrated onto the silicon photonics platform. This accomplishes two outstanding difficulties with exploiting nanofocusing in modern technology. Firstly, we have managed to demonstrate nanophotonic waveguides with lateral dimensions down to $24 \mathrm{~nm}$ over distances of about 10 microns using electron beam lithography. This represents optical confinement at least 1/100th of the area of typical optical waveguide used in silicon photonics. Secondly, we are able to couple light from a long range photonic-like silicon waveguide mode directly into these tightly localized plasmonic modes by simply varying one geometrical parameter of the lithography. This provides us with a robust method to achieve efficient "adiabatic" nanofocusing, in this case, a $167 \pm 26$ electric field intensity enhancement for a $24 \mathrm{~nm}$ wide plasmonic waveguide.

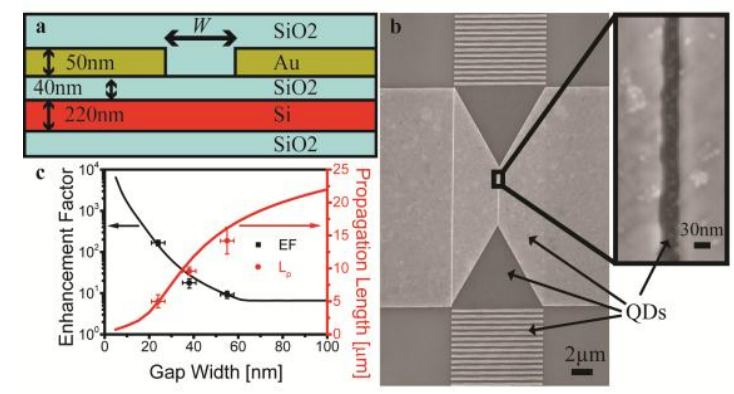

